\title{
Registry of 919 Patients with Thrombotic Microangiopathies across Japan: Database of Nara Medical University during 1998-2008
}

\author{
Yoshihiro Fujimura and Masanori Matsumoto
}

\begin{abstract}
Background Thrombotic microangiopathies (TMAs) are pathological conditions characterized by generalized microvascular occlusion by platelet thrombi, thrombocytopenia, and microangiopathic hemolytic anemia. Two typical phenotypes of TMAs are hemolytic-uremic syndrome (HUS) and thrombotic thrombocytopenic purpura (TTP). Severe deficiency of plasma ADAMTS13 activity (ADAMTS13: AC) is more specific for TTP, but not for HUS. Since 1998, our laboratory has functioned as a nationwide referral center for TMAs by analyzing ADAMTS13.

Methods Of 1,564 patients tested from 426 hospitals, 919 were positive for TMA. Levels of ADAMTS13: AC and the ADAMTS13 neutralizing autoantibody (ADAMTS13: INH) were determined by chromogenic act-ELISA and/or by classic von Willebrand factor multimer assay.

Results TMA patients consisted of two groups: severe (less than 3\% of normal control) and non-severe deficiency of ADAMTS13: AC. Both groups were divided into congenital $(n=65)$ and acquired $(n=854)$ TMA. Of the former, 41 had congenital deficiency of ADAMTS13: AC, while the remaining 24 had disease of unknown etiology. The 854 patients with acquired TMA could be largely grouped into three categories: idiopathic TTP $(n=284)$, idiopathic HUS $(n=106)$, and secondary TMAs $(n=464)$. The secondary TMAs were observed in heterogeneous patient groups and were associated with drugs, connective tissue diseases, malignancies, transplantation, pregnancy, E. coli O157: $\mathrm{H} 7$ infection, and other factors. All of the patients with acquired severe ADAMTS13: AC deficiency were positive for ADAMTS13: INH.

Conclusion Although TMAs are highly heterogeneous pathological conditions, one-third of TMA patients have severe deficiency of ADAMTS13: AC. Platelet transfusions to such patients are contraindicated. Rapid ADAMTS13: AC assays are therefore prerequisite to appropriately treat TMA patients.
\end{abstract}

Key words: TMA, TTP, HUS, USS, ADAMTS13, VWF

(Inter Med 49: 7-15, 2010)

(DOI: 10.2169/internalmedicine.49.2706)

\section{Introduction}

Thrombotic microangiopathies (TMAs) are pathological conditions that are characterized by microangiopathic hemolytic anemia, vast microvascular occlusions caused by platelet thrombi (common renal involvement), and thrombocytopenia (1). Two typical phenotypes of TMAs are hemolytic-uremic syndrome (HUS) and thrombotic thrombocytopenic purpura (TTP), both of which are life-threatening diseases. HUS is characterized by the aforementioned three clinical signs (classic 'triad'), while TTP is characterized by a classic 'pentad,' which includes the 'triad' as well as fever and neurological signs; however, the two diseases are often indistinguishable. Further, these TMAs must be differentiated from disseminated intravascular coagulation (DIC) or consumptive thrombohemorrhagic disorders (2).

In 1996, a metalloprotease that specifically cleaves von Willebrand factor (VWF) was identified in normal plasma $(3,4)$, and 5 years later this enzyme was purified, 
cloned, and termed ADAMTS13 (a disintegrin-like metalloproteinase with thrombospondin type 1 motifs 13) (5-8). ADAMTS13-producing cells were initially identified within the liver, and then more specifically as hepatic stellate cells (9), but now it is known that ADAMTS13 is also present in platelets (10), vascular endothelial cells (11), and kidney podocytes (12). Since the discovery of ADAMTS13, severe deficiency of ADAMTS13 activity (ADAMTS13: AC) has been thought to be a unique feature of TTP, and can be caused by genetic mutations or by acquired autoantibody (ADAMTS13: INH) to this enzyme; however, these alterations are not observed in HUS patients $(13,14)$. It is notable that a minor population of TTP patients with the "pentad' of symptoms has almost normal or only slightly reduced ADAMTS13: AC (15). In this regard, Tandon et al (16) reported in 1994 that approximately 80\% of the patients with acquired TTP had anautoantibody against CD36. In those days, however, this finding could not be directly linked to the pathogenesis of TTP. Recently, Davis et al (17) have shown that recombinant (r)-human ADAMTS13 specifically binds to r-human CD36 in vitro. CD36 is expressed in endothelial cells, platelets, and monocytes, and has been reported to bind thrombospondin-1 (18). ADAMTS13, after secretion into the circulation, is assumed to efficiently cleave unusually large VWF multimers (UL-VWFMs) released from vascular endothelial cells as a solid-phase enzyme by binding to the cell surface. It is currently unclear whether anti-CD36 autoantibodies block ADAMTS13 binding to vascular endothelial cells, but if so, this may interfere with the efficient cleavage of UL-VWFMs by ADAMTS13 and result in TTP.

In contrast to TTP, HUS is rarely induced by genetic mutations in complement regulatory factors (factors $\mathrm{B}, \mathrm{H}$, and $\mathrm{I}$, and membrane cofactor protein or CD46). HUS can also be acquired, typically following acute enterocolitis due to shigatoxin-producing Echerichia coli O157: H7 infection, but also rarely due to autoantibody against factor $H$ (19).

Since 1998, our laboratory at Nara Medical University has functioned as a nation-wide referral center for TMAs via assaying ADAMTS13: AC in a large Japan-wide patient population with thrombocytopenia suspected of being TMA. As of the end of 2008, we have established a registry of 919 patients with TMAs, and have analyzed their clinical and laboratory information. Here, we describe the results of this study, and discuss the divergence of TMAs among patient groups with masked or unmasked thrombocytopenia.

\section{Materials and Methods}

\section{Patients}

Between July 1998 and December 2008, plasma samples from 1,564 patients with thrombocytopenia suspected of TMAs were referred to our laboratory with clinical and laboratory information from 426 medical institutions across Japan. All subjects provided informed consent to participate in this study. The study protocol was approved by the Ethics Committee of Nara Medical University Hospital.

\section{Blood sampling}

Before therapeutic approaches including plasma infusion, plasma exchange, and the use of immunosuppressants, whole blood samples $(-5 \mathrm{~mL})$ were taken from each patient into plastic tubes containing $1 / 10$ volume of $3.8 \%$ sodium citrate. The plasma was separated by centrifugation at 3,000 $\times \mathrm{g}$ for $15 \mathrm{~min}$ at $4{ }^{\circ} \mathrm{C}$, kept in aliquots at $-80^{\circ} \mathrm{C}$ until testing, and sent to our laboratory.

\section{Assays of plasma ADAMTS13: AC and ADAMTS13: INH}

Until March 2005, ADAMTS13: AC was determined by classic VWFM assay (3) with a detection limit of $3 \%$ of the normal control (20). Thereafter, a chromogenic ADAMTS 13-act-ELISA with a detection limit of $0.5 \%$ of the normal control was developed (21), and replaced the VWFM assay. Measurement of plasma levels of ADAMTS13: AC by these assays were highly correlated $\left(\mathrm{R}^{2}=0.72, \mathrm{p}<0.01\right)$ and provided similar results for mean $\pm \mathrm{SD}$ in healthy individuals $(102.4 \pm 23.0 \%$ vs. $99.1 \pm 21.5 \%)$, as shown previously $(21,22)$. Thus, we re-examined the plasma of 724 of the 774 TMA patients determined by the VWFM assay by act-ELISA, and the latter data were used in this study. For 50 TMA patients we were unable to re-examine by actELISA, the VWFM assay data were used. We have therefore tentatively categorized plasma levels of ADAMTS13: AC of $<3 \%, 3 \%-<25 \%$, and $25 \%-<50 \%$ of the normal as severe, moderate, and mild deficiency, respectively.

Plasma ADAMTS13: INH titers were also evaluated either by classic VWFM assay or chromogenic ADAMTS13-actELISA using heat-inactivated plasma at $56^{\circ} \mathrm{C}$ for 30 minutes $(13,14)$. One Bethesda unit $(U)$ is defined as the amount necessary to reduce ADAMTS13: AC to 50\% of control levels (23). Titers greater than 0.5 Bethesda U/mL were classified as inhibitor positive.

\section{Diagnostic criteria for TMAs}

According to previous reports $(2,24,25)$, TMAs were defined as having all of the following: (i) microangiopathic hemolytic anemia (hemoglobin $\leq 12 \mathrm{~g} / \mathrm{dL}$ ), Coombs test negative, undetectable serum haptoglobin $(<10 \mathrm{mg} / \mathrm{dL})$, more than 2 fragmented red cells (schistocytes) in a microscopic field with a magnification of 100, and concurrent increased serum lactate dehydrogenase (LDH) above institutional baseline; (ii) thrombocytopenia (platelet count $\leq 100 \times$ $10^{9} / \mathrm{L}$ ); and (iii) a variable severity of organ dysfunction (renal or neurological involvement) devoid of the stigmata of DIC (26).

A differential diagnosis of HUS or TTP based on routine laboratory data is usually difficult. As a rule, plasma levels of ADAMTS13: AC were first determined on all patients suspected of TMAs, and patients with severe deficiency of ADAMTS13: AC were classified as TTP regardless of clini- 


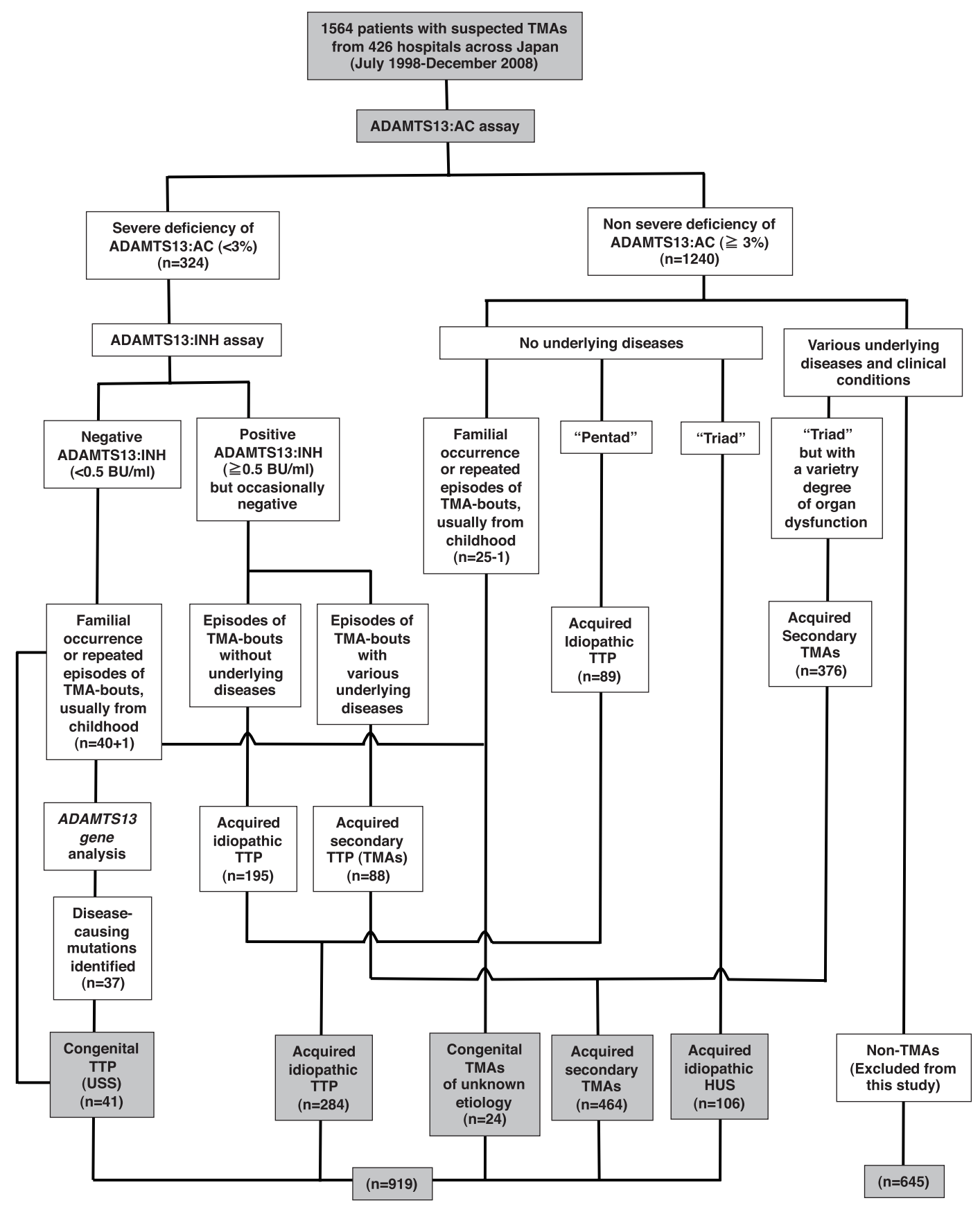

Figure 1. Flow chart of categorization of patients with suspected thrombotic microangiopathies (TMAs) based on ADAMTS13 analysis. Of 1,564 patients with suspected TMAs, 324 had severe deficiency of ADAMTS13 activity and 1,240 did not. In the former category, 40 patients were categorized as USS and 284 as acquired TTP. In the latter category, 24 patients were categorized as congenital TMAs of the unknown etiology, 570 as acquired TMAs, and one patient as USS with moderately reduced plasma ADAMTS13:AC (3.4\%), to whom frequent plasma infusions had been made to prevent further aggravation of cerebral infarction. The remaining 645 patients did not have TMAs and were therefore excluded from this study.

cal signs. Second, the patients were grouped as HUS or TTP based on the 'triad' or 'pentad' of clinical signs. This protocol appeared to be important, because our registry includes patients with hereditary deficiency of ADAMTS13: AC or congenital TTP (Upshaw-Schulman syndrome, USS), which generally have less severe clinical signs (often isolated thrombocytopenia) than acquired TTP.

\section{Results and Discussion}

A flow chart of patient categorization based on ADAMTS 13 analysis is shown in Fig. 1. Of the 1,564 patients referred to our laboratory, 324 (minor population) had severe deficiency of ADAMTS13: AC and 1,240 (major population) did not. In the population with severe ADAMTS13: AC de- 
Table 1. Plasma Levels of ADAMTS13: AC and ADAMTS13: INH in 919 Patients with Thrombotic Microangiopathies (TMAs) Registered at Nara Medical University during July 1988- December 2008

\begin{tabular}{|c|c|c|c|c|c|c|c|c|c|c|c|c|c|c|}
\hline & \multicolumn{2}{|c|}{ Congenital TMAs } & \multicolumn{11}{|c|}{ Acquired TMAs } & \multirow{4}{*}{$\begin{array}{c}\text { Total } \\
(n=919)\end{array}$} \\
\hline & \multirow{3}{*}{$\begin{array}{l}\text { Upshaw- } \\
\text { Schulman } \\
\text { syndrome } \\
\text { (USS) } \\
(n=41)\end{array}$} & \multirow{3}{*}{\begin{tabular}{|} 
Unknown \\
etiology \\
$(n=24)$
\end{tabular}} & \multicolumn{2}{|c|}{ Idiopathic } & \multicolumn{9}{|c|}{ Secondary } & \\
\hline & & & \multirow{2}{*}{$\begin{array}{c}\text { Thrombotic } \\
\text { thrombocytopenic } \\
\text { purpurar (TTP) } \\
(\mathrm{n}=284)\end{array}$} & \multirow{2}{*}{$\begin{array}{c}\text { Hemolytic- } \\
\text { uremic } \\
\text { syndroime } \\
\text { (HUS) } \\
(n=106)\end{array}$} & \multicolumn{3}{|c|}{ Drug-induced } & \multirow{2}{*}{$\begin{array}{c}\text { Conective tissue } \\
\text { diseases and } \\
\text { their allied } \\
\text { diseases } \\
\text { (CTDs/ADs) } \\
\text { (n=221) }\end{array}$} & \multirow[b]{2}{*}{$\underset{(n=61)}{\text { Malignancies }}$} & \multirow{2}{*}{$\begin{array}{c}\text { Hematopoietic } \\
\text { stem cell } \\
\text { transplant- } \\
\text { ation } \\
\text { (HSCT) } \\
(n=54)\end{array}$} & \multirow[b]{2}{*}{$\underset{(\mathrm{n}=15)}{\text { Pregnancy }}$} & \multirow[b]{2}{*}{$\begin{array}{c}\text { E. coli } \\
\text { O157: } \mathrm{H7} \\
\text { infection } \\
(\mathrm{n}=32)\end{array}$} & \multirow[b]{2}{*}{$\begin{array}{c}\text { Others } \\
\text { (Liver } \\
\text { cirrhosis, } \\
\text { etc) }(n=46)\end{array}$} & \\
\hline & & & & & $\begin{array}{c}\text { Ticlopidine } \\
(n=2) / \\
\text { Clopidogrel } \\
(n=1)\end{array}$ & $\underset{(\mathrm{n}=10)}{\operatorname{Mitomycin} \mathrm{C}}$ & $\begin{array}{c}\text { Pegylated- } \\
\text { interferon }(n=1) \\
\text { / Sildenafil }(n=1)\end{array}$ & & & & & & & \\
\hline $\begin{array}{c}\underset{(\%)}{\operatorname{ADAMTS} 13: A C} \\
.\end{array}$ & $(n=41)$ & $(n=24)$ & $(n=284)$ & $(n=106)$ & $(n=22 / n=1)$ & $(n=10)$ & $(n=1 / n=1)$ & $(n=221)$ & $(n=61)$ & $(n=54)$ & $(n=15)$ & $(n=32)$ & $(n=46)$ & ( $\mathrm{n}=919)$ \\
\hline$<3$ & 40 & 0 & 195 & 0 & 19 & 0 & 2 & 46 & 5 & 0 & 4 & 0 & 13 & 324 \\
\hline $3 \sim<25$ & 1 & 4 & 72 & 20 & 2 & 2 & 0 & 66 & 23 & 18 & 4 & 5 & 16 & 233 \\
\hline $25 \sim<50$ & 0 & 9 & 14 & 48 & 1 & 5 & 0 & 66 & 22 & 24 & 4 & 17 & 6 & 216 \\
\hline$\geqq 50$ & 0 & 11 & 3 & 38 & 1 & 3 & 0 & 43 & 11 & 12 & 3 & 10 & 11 & 146 \\
\hline $\begin{array}{c}\text { ADAMTS13:INH } \\
(\mathrm{U} / \mathrm{ml})\end{array}$ & $(n=41)$ & $(n=23)$ & $(n=282)$ & $(n=43)$ & $(n=22 / n=1)$ & $(n=7)$ & $(n=1 / n=1)$ & $(n=187)$ & $(n=26)$ & $(n=15)$ & $(n=8)$ & $(n=17)$ & $(n=23)$ & ( $n=697)$ \\
\hline $0.5 \sim<2$ & 0 & 0 & 129 & 2 & 6 & 0 & 2 & 80 & 8 & 4 & 2 & 1 & 8 & 242 \\
\hline$<0.5$ & 41 & 23 & 33 & 41 & 2 & 7 & 0 & 79 & 13 & 11 & 3 & 16 & 6 & 275 \\
\hline
\end{tabular}

() Sample number determined

ficiency, 40 patients were categorized as USS and 284 as acquired TTP, and no patients with DIC or septic DIC were included. In the population without severe ADAMTS13: AC deficiency, 24 patients were categorized as congenital TMAs of unknown etiology, 570 as acquired TMAs, and only one patient (GG in Table 2) as USS with moderately reduced plasma ADAMTS13: AC (3.4\%), to whom frequent plasma infusions had been made to prevent further aggravation of cerebral infarction. Thus, a diagnosis of USS in this patient GG was made after identifying the disease-causing mutations $(\mathrm{C} 1024 \mathrm{R} / \mathrm{C} 1024 \mathrm{R})$ in exon 24 by ADAMTS13 gene analysis. These data will be published elsewhere in detail. The remaining 645 patients did not have TMAs, and were therefore excluded from this study; this group included 64 patients with DIC or septic DIC.

\section{Congenital TMAs}

Patients with repeated TMA episodes usually starting in early childhood with or without familial occurrence are usually considered as congenital TMAs; these patients are largely separated into the following two categories, on the basis of plasma levels of ADAMTS13: AC and ADAMTS 13: INH.

\section{Upshaw-Schulman syndrome (USS)}

USS is alternatively termed congenital TTP and is characterized by severe deficiency of ADAMTS13: AC due to genetic mutations (27). Forty-one patients (25 females and 16 males) belonging to 36 different families, were placed in this category (Table 2). All of these patients were negative for ADAMTS13: INH. USS is inherited in an autosomal recessive fashion, and therefore, the parents of patients are asymptomatic carriers with significantly reduced plasma levels of ADAMTS13: AC. The female-to-male ratio in the USS patient population is theoretically one-to-one, but our results indicate an apparent female predominance (25 to 16). Of the 41 patients, $17(41 \%)$ had a history of exchange blood transfusions during the newborn period, and 32 (78\%) had a history of thrombocytopenia during childhood. For the remaining $9(22 \%)$, it was unclear whether their platelet counts had been checked during that period.

ADAMTS13 gene analysis was performed for 38 USS patients, and the disease-causing mutations were identified in 37 of the 38 . Of the 37 genotyped patients, 8 were homozygotes and 29 were compound heterozygotes [one de novo mutation (28)] for ADAMTS13 gene mutations. Of the 8 homozygous patients, the parents of 6 had consanguineous marriages.

\section{Congenital TMAs of unknown etiology}

Patients in this category were characterized by repeated TMA episodes with predominant renal involvement from early childhood, and often with familial occurrence. Twentyfour patients belonging to 12 families were identified, but the etiology of TMAs in these patients remained completely unclear.

In this regard, it is well known that gene mutations in complement regulatory cofactors (factor $\mathrm{H}$, factor $\mathrm{I}$, factor $\mathrm{B}$, and CD46 or membrane cofactor protein) cause excessive complement activation by impairing $\mathrm{C} 3 \mathrm{~b}$ inactivation, resulting in severe hemolysis, which triggers TMA episodes. Therefore, these patients are commonly termed 'congenital atypical HUS' (19). It is possible that among the patients of this category in this study, some disease might be related to gene mutations of complement regulatory cofactors, but at the time such analysis had not been done in Japan. As a first step toward such analysis, we determined the plasma levels of factor $\mathrm{H}$ antigen by immunoassay in our patients, and did not observe reduced levels in any patients (data not shown). 
Table 2. Registration of 41 Japanese Patient with Upshaw-Schulman Syndrome (USS)

\begin{tabular}{|c|c|c|c|c|c|c|c|}
\hline No & Patient & $\begin{array}{c}\text { Year } \\
\text { of } \\
\text { birth }\end{array}$ & Sex & $\begin{array}{l}\text { Exchange blood } \\
\text { transfusion } \\
\text { during newborn } \\
\text { period }\end{array}$ & $\begin{array}{l}\text { Thrombocytopenia } \\
\text { during childdhood }\end{array}$ & $\begin{array}{c}\text { Plasma } \\
\text { ADAMTS13:AC } \\
(\%)\end{array}$ & $\begin{array}{c}\text { ADAMTS13 } \\
\text { gene mutations }\end{array}$ \\
\hline 1 & A & 1999 & M & + & + & $<0.5$ & C-Hetero \\
\hline 2 & B & 1986 & $\mathbf{F}$ & + & + & $<0.5$ & Homo \\
\hline 3 & c & 1972 & M & - & + & $<0.5$ & Homo \\
\hline 4 & D & 1978 & $\mathbf{F}$ & + & + & $<0.5$ & C-Hetero \\
\hline 5 & E & 1985 & M & + & + & $<0.5$ & C-Hetero \\
\hline 6 & $\mathbf{F}$ & 1993 & M & + & + & 0.6 & C-Hetero \\
\hline 7 & G & 1987 & $\mathbf{F}$ & + & + & $<0.5$ & C-Hetero \\
\hline 8 & H & 1951 & M & - & - & 0.6 & C-Hetero \\
\hline 9 & 1 & 1972 & M & - & + & $<0.5$ & C-Hetero \\
\hline 10 & $\mathrm{~J}-3$ & 1977 & $\mathbf{F}$ & - & + & $<0.5$ & C-Hetero \\
\hline 11 & J-4 & 1979 & M & - & + & $<0.5$ & C-Hetero \\
\hline 12 & $K-3$ & 1976 & $\mathbf{F}$ & - & + & $<0.5$ & C-Hetero \\
\hline 13 & K-4 & 1978 & $\mathbf{F}$ & + & + & $<0.5$ & C-Hetero \\
\hline 14 & L-2 & 1967 & $\mathbf{F}$ & $\cdot$ & - & $<0.5$ & C-Hetero \\
\hline 15 & $\mathrm{~L}-3$ & 1972 & $\mathbf{F}$ & - & + & $<0.5$ & C-Hetero \\
\hline 16 & M-3 & 1969 & $\mathbf{F}$ & - & - & $<0.5$ & C-Hetero \\
\hline 17 & M-4 & 1971 & $\mathbf{F}$ & - & - & $<0.5$ & C-Hetero \\
\hline 18 & $\mathbf{N}$ & 1986 & $\mathbf{F}$ & + & + & $<0.5$ & C-Hetero \\
\hline 19 & $0-4$ & 1958 & $\mathbf{F}$ & - & - & $<0.5$ & C-Hetero \\
\hline 20 & $P$ & 1971 & M & $\cdot$ & + & $<0.5$ & C-Hetero \\
\hline 21 & $Q(1)$ & 1983 & M & + & + & $<0.5$ & C-Hetero \\
\hline 22 & $Q(2)$ & 1988 & M & + & + & $<0.5$ & C-Hetero \\
\hline 23 & R-5 & 1982 & $\mathbf{F}$ & - & + & $<0.5$ & C-Hetero \\
\hline 24 & s & 1982 & $\mathrm{~F}$ & - & + & 0.9 & $*$ \\
\hline 25 & T & 1981 & F & - & + & $<0.5$ & C-Hetero \\
\hline 26 & u & 1990 & $\mathbf{F}$ & + & + & $<0.5$ & Homo \\
\hline 27 & v & 1983 & $\mathbf{F}$ & + & + & $<0.5$ & C-Hetero \\
\hline 28 & W-4 & 1990 & $\mathbf{F}$ & - & + & $<0.5$ & C-Hetero \\
\hline 29 & $X-5$ & 1963 & $\mathbf{F}$ & - & - & $<0.5$ & $*$ \\
\hline 30 & $\mathbf{Y}$ & 1960 & $\mathbf{F}$ & - & + & $<0.5$ & C-Hetero \\
\hline 31 & Z-3 & 1971 & $\mathbf{F}$ & - & + & $<0.5$ & Homo \\
\hline 32 & AA & 1987 & $\mathbf{F}$ & - & - & $<0.5$ & $*$ \\
\hline 33 & BB & 1947 & M & - & - & $<0.5$ & Homo \\
\hline 34 & CC-5 & 2004 & M & + & + & $<0.5$ & C-Hetero \\
\hline 35 & DD & 2007 & $\mathbf{F}$ & - & + & $<0.5$ & C-Hetero \\
\hline 36 & $\mathrm{EE}$ & 2003 & M & + & + & $<0.5$ & Homo \\
\hline 37 & FF & 1991 & $\mathbf{F}$ & + & + & $<0.5$ & Homo \\
\hline 38 & GG & 1931 & M & - & - & 3.4 & Homo \\
\hline 39 & $\mathrm{HH}$ & 2004 & $\mathbf{F}$ & + & + & $<0.5$ & C-Hetero \\
\hline 40 & II & 1977 & $\mathbf{F}$ & + & + & $<0.5$ & $*$ \\
\hline 41 & JJ & 1977 & M & - & + & $<0.5$ & C-Hetero \\
\hline
\end{tabular}

C-Hetero: Compound heterozygotes, Homo: Homozygotes, *: Not determined.

\section{Acquired TMAs}

Patients with acquired TMAs are characterized by the following: 1) usually no familial occurrence, 2) presence or absence of underlying diseases or medications associated with TMAs, and 3) common sudden onset of TMA episodes during adulthood. Patients with acquired TMAs are grouped as primary (idiopathic) or secondary, and then further separated into categories as follows, based on the results of ADAMTS 13: AC and ADAMTS13: INH assays.

\section{Idiopathic TMAs}

The patients in this group lack apparent underlying diseases or medications related to TMA episodes. Idiopathic TMAs can be further categorized into TTP and HUS subgroups. Idiopathic TTP $(\mathrm{n}=284)$ included two patient populations: 1) patients $(n=195)$ with severe deficiency of ADAMTS13: AC, commonly positive for ADAMTS13: INH, and 2) patients $(n=89)$ with clinical 'pentad' signs, regardless of plasma ADAMTS13: AC levels. Distribution of plasma ADAMTS13: AC is shown in Table 1. Detailed analysis of the clinical and laboratory features of these pa- tients will be published elsewhere.

In contrast, idiopathic HUS $(n=106)$ consisted of one patient population with clinical 'triad' signs, without severe deficiency of ADAMTS13: AC. Two patients of this category exhibited low levels of ADAMTS13: INH $(0.5-<2$ BU/ $\mathrm{mL})$.

\section{Secondary TMAs}

Secondary TMAs develop in the setting of various clinical conditions, such as infection, medication, and various underlying diseases. For instance, acquired TMAs are often associated with connective tissue diseases, and also treatment using several specific drugs. In these patients, clinical signs are often highly variable, so diagnostic differentiation of TTP or HUS appears to be insignificant.

\section{(1) Drug-induced TMAs}

A significant number of drugs have been associated with TMAs, including anti-platelet thienopyridine derivative drugs, antineoplastic drugs such as mitomycin $\mathrm{C}$, and quinine (29). We have no experience with quinine-associated TMAs, but observed two suspected drug-associated TMAs: 
one with sildenafil (Viagra) and the other with pegylatedinterferon. Thus, drug-induced TMAs will be discussed in the following 3 subgroups.

\section{a) Thienopyridine derivative-induced TMAs}

Ticlopidine (TC) and clopidogrel (CL) are two typical thienopyridine derivatives (30). We identified 22 patients with TC-induced TMAs and one with CL-induced TMA. Nineteen of the 22 patients with TC-TMAs (86\%) had severe ADAMTS13: AC deficiency and were positive for ADAMTS13: INH. The mechanism by which TC induces TMAs is still unclear, but it is speculated that TC becomes active in circulation and binds to ADAMTS13, forming a hapten-carrier complex. Antibodies formed against such a complex may be specific for the hapten, the combination hapten-carrier site, or the carrier alone, in a similar fashion to alpha-methyldopa, which may cause the development of anti-red cell antibodies. In approximately $90 \%$ of patients with TC-induced TMAs, the onset of TMA episodes occurred within 40 days of treatment (30). The frequency of TC-induced TMAs is estimated to be one per 1,600 to 5,000 patients. In contrast, only one female patient with CLinduced TMA, who developed TMA episodes 4 days after treatment, has been reported in Japan (31). This patient had slightly reduced plasma ADAMTS13: AC (34\%), and was negative for ADAMTS13: INH. The pathogenesis of CLinduced TMAs is unclear, but recent studies suggest that ADAMTS13 is released from the liver into circulation, binds to endothelial cell surfaces, and efficiently cleaves ULVWFMs. Thus, if endothelial cell injuries are present, ADAMTS13 cannot effectively cleave UL-VWFMs; this may lead to TMA episodes. In this regard, Zakarija et al (32) recently addressed two mechanistic pathways in TMAs related to thienopyridine derivatives.

\section{b) Mitomycin C-induced TMAs}

Ten patients with mitomycin C (MMC)-induced TMAs were identified. None had severe deficiency of ADAMTS13: $\mathrm{AC}$, and all were negative for ADAMTS13: INH. Previous reports (33) suggest that MMC-induced TMAs develop with a frequency of $4-15 \%$ of the patients treated with this drug. The pathophysiology of MMC-TMAs is not well understood, but it is assumed that MMC may cause vascular endothelial cell injuries.

\section{c) TMAs associated with other drugs}

We observed two other TMA patients with severe deficiency of ADAMTS13: AC and positive ADAMTS13: INH. Both of these patients were assumed to have drug-associated TMA. One patient was a 62-year-old male with chronic hepatitis C. This patient developed TMA a month after longterm treatment with pegylated-interferon; the detailed clinical course of this patient was previously reported (34). The other patient with possible drug-induced TMA was a 65year-old male who had taken sildenafil. The patient had taken sildenafil once several months prior to development of
TMA, and then he had taken the drug twice within the 2 weeks prior to TMA. Two days after his third intake of sildenafil, the patient developed a low-grade fever, hemolytic anemia (hemoglobin $10.3 \mathrm{~g} / \mathrm{dL}$ and reticulocyte $3.9 \%$ ), thrombocytopenia $(11,000 / \mu \mathrm{L})$, and hematuria. ADAMTS 13 analysis identified severe deficiency of ADAMTS13: AC $(<3 \%)$ and ADAMTS13: INH positivity (1.5 Bethesda U/ $\mathrm{mL})$. The patient was treated by oral administration of the anti-platelet drug dipyridamole without plasma exchange. Since then, he has recovered, and his ADAMTS13: AC returned to normal range 3 months later.

\section{(2) Connective tissue diseases and their allied dis- eases (CTD/AD)-associated TMAs}

A close relationship between systemic lupus erythematosus (SLE) and TTP was first described in 1939 (35). It is now known that TMAs are frequently associated with CTDs with a frequency of $1-6 \%$ of the patient population (36). We have recently reported that severe deficiency of ADAMTS 13: AC and positive ADAMTS13: INH was predominantly detected in patients with rheumatoid arthritis (RA)- and SLE-associated TMAs, via the analysis of 127 patients with CTD-associated TMAs, whose samples were collected between 1998-2006 (37).

In this study, we included other miscellaneous autoimmune diseases, such as antiphospholipid syndrome (APS), as listed in Table 3, in the analysis. Thus, we examined 221 patients with CTD/AD-associated TMAs (Tables 1, 3), of whom $46(21 \%)$ had severe deficiency of ADAMTS13: AC with positive ADAMTS13: INH, while the remaining 175 (79\%) had mild-to-moderate deficiency. We presume that the high prevalence of TMA episodes in patients with CTD/AD is closely related to high plasma levels of VWF over the low levels of ADAMTS13: AC (37). Anatomical changes of the microvasculature, namely narrowed vessel cavities due to the proliferation of vascular endothelial cells, result in altered circulation hemodynamics and contribute to the formation of platelet thrombi at sites of vascular injury.

\section{(3) Malignancy-associated TMAs}

Sixty-one patients were classified into this category, which largely consisted of 2 groups: one group of patients with hematological malignancies $(n=30)$ and the other group with malignant solid tumors $(n=31)$ (Table 2).

Of the hematological malignancies, lymphoma was the most frequently seen $(n=16)$, and four of the 16 patients had severe deficiency of ADAMTS13: AC with positive ADAMTS13: INH. The clinical course of one patient with intravascular lymphoma (IVL)-associated TMA was previously reported (38). In this case, the aggravation of TMA was dependent on the treatment efficacy of chemotherapy during the early stage of disease progression, but in the later stage was dependent on rituximab after several relapses during a 4-year observation period (39).

Of 31 patients with malignant solid tumor-associated TMAs, stomach cancer $(n=10)$ was most commonly seen, 
Table 3. Details of Underlying Diseases or Clinical Conditions of Patients with Acquired Secondary Thrombotic Microangiopathies (TMAs)

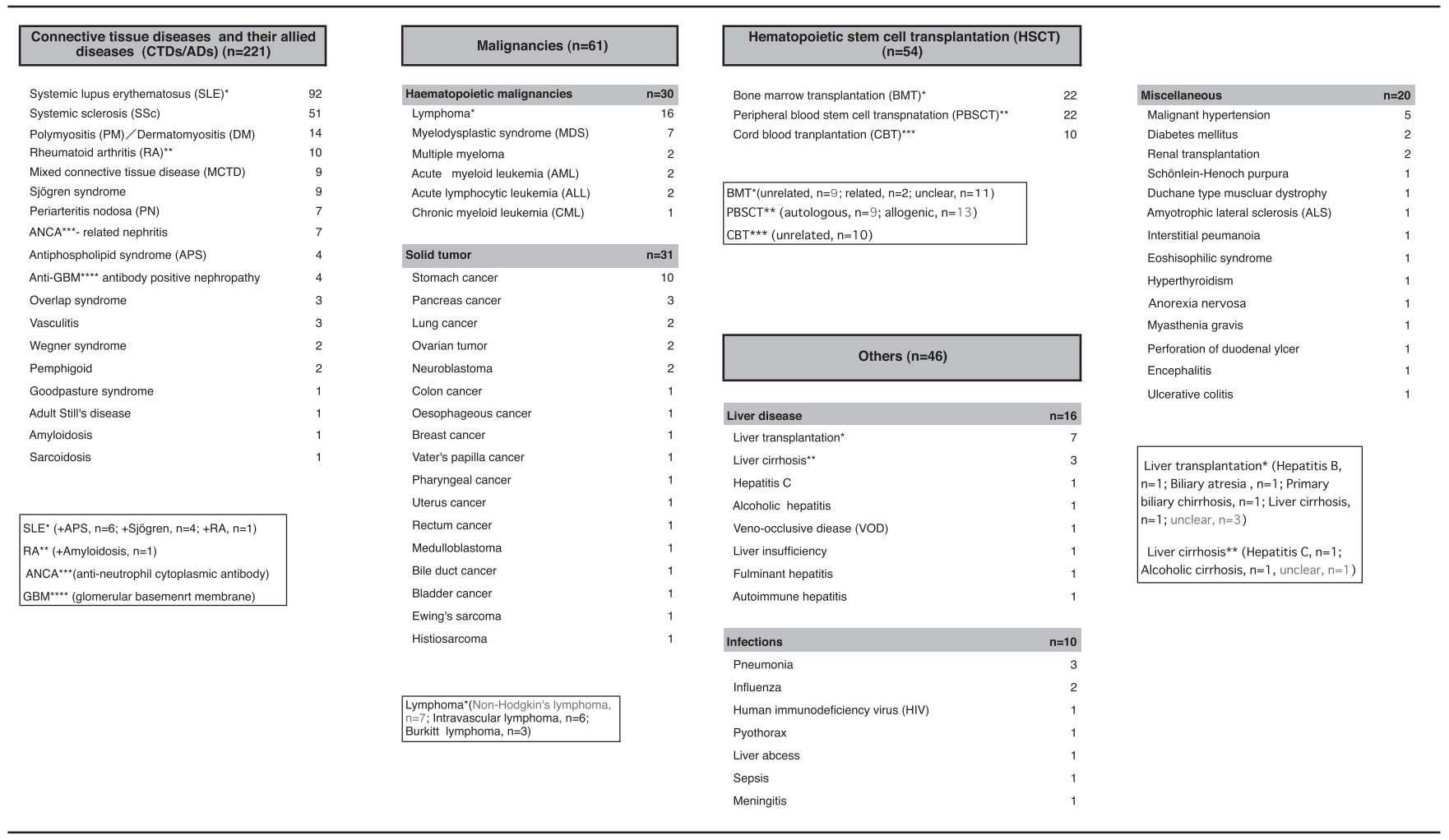

but a variety of organs were involved as listed in Table 3. One patient with Vater's papilla cancer showed severe deficiency of ADAMTS13: AC with the presence of ADAMTS 13: INH.

\section{(4) Hematopoietic stem cell transplantation (HSCT)- associated TMAs}

Fifty-four patients with TMAs were classified in this category. Of these, 22 were associated with bone marrow transplantation, 22 with peripheral blood SCT, and 10 with cord blood SCT (Table 3). The pathogenesis of TMAs in this category is highly complicated by pre-conditioning regimens of chemotherapies and body irradiation, as well as posttransplantation complications, such as bacterial or viral infections and graft-versus-host disease (GVHD). It was remarkable that none of the patients in this category had severe deficiency of ADAMTS13: AC, and all were negative for ADAMTS13: INH, as previously reported by others (40).

\section{(5) Pregnancy-associated TMAs}

TMA episodes are sometimes precipitated by pregnancy and postpartum, and require a rapid differential diagnosis from other thrombocytopenic status, such as ITP, pregnancy toxemia, eclampsia, and HELLP (hemolysis, elevated liver enzymes, and low platelet count) syndrome. The category of pregnancy-associated TMAs was present in two clinical settings: USS (congenital TTP) and acquired TTP. We have recently reported that pregnancy induced isolated thrombocytopenia or TTP in all female patients with USS examined, and their babies were stillborn or premature when the appro- priate plasma therapy was not performed (41).

In the present study, we were able to identify 15 patients with acquired TMAs associated with pregnancy or postpartum. Of these, eight patients developed TMA episodes at 10-40 weeks of gestation, six patients developed TMA episodes soon after delivery, and one patient developed TMA episodes 3 months postpartum. Four of the 15 patients (27\%) had severe deficiency of ADAMTS13: AC with positive ADAMTS13: INH (Table 1). These results suggest that the pathogenesis of acquired pregnancy-associated TMAs may be multifactorial, a different setting from USS.

\section{(6) Escherichia coli 0157 H7-associated TMAs:}

Shigatoxin (1 and 2), composed of one A-subunit of 33 $\mathrm{kDa}$ and five $\mathrm{B}$-subunits of $7 \mathrm{kDa}$ each, is produced by the E. coli O157: H7 strain. Shigatoxin binds to a receptor, termed globotriaosyl ceramide, which is richly expressed in glomerular endothelial cells. After binding, shigatoxin is internalized and it induces endothelial cell apoptosis; this releases significant levels of UL-VWFMs into the circulation, resulting in platelet thrombi within microvasculatures. Hence, E. coli O157: H7-associated TMAs appear to be induced independent of plasma levels of ADAMTS13: AC. Thirty-two patients with TMAs were in this category, and in fact none of them had severe deficiency of ADAMTS13: AC. However, 22 patients had slightly reduced ADAMTS13: AC (Table 1). The reason underlying this is unclear, but we postulate either that ADAMTS13 is partially consumed to cleave the increased plasma VWF or that shigatoxin directly targets ADAMTS13-producing cells. 


\section{(7) TMAs associated with other causes}

Forty-six TMA patients, who did not fit the aforementioned categories, were classified in this category (Table 3). Because of high heterogeneity in this category, it was subcategorized into patients with liver diseases $(n=16)$, those with infections $(n=10)$, and miscellaneous causes $(n=20)$.

We have reported that numerous liver diseases are associated with reduced plasma ADAMTS13: AC. Notably, plasma levels of ADAMTS13: AC decline in parallel to the progression of liver cirrhosis (42). More interestingly, several patients with advanced liver cirrhosis had severe deficiency of ADAMTS13: AC with positive ADAMTS13: INH. These patients were assumed to have cryptic clinical signs of TMA; therefore, the term 'subclinical TTP' was introduced. In addition, we have reported on recipients of liver transplants with early allograft dysfunction who showed severe thrombocytopenia accompanied by a marked reduction of ADAMTS13: AC one or two days after transplantation, but without any apparent clinical features of TMAs (43). This observation has been confirmed by two recent reports $(44,45)$, but the mechanism has not yet been addressed.

Viral or bacterial infections can trigger TMA episodes, but the mechanism has not yet been addressed. Most recently, influenza has been revisited by researchers, due to a close relationship between influenza and TMA originally reported in 1980 (46). It is now known that influenza vaccine may induce TTP or disease relapse (47). We have two patients with influenza A-associated TMAs, and one of them had severe deficiency of ADAMTS13: AC with positive ADAMTS13: INH. Influenza virus or vaccination often worsens underlying diseases or conditions, including diabetes mellitus, pregnancy, and ongoing hemodialysis, resulting in multiorgan failure (MOF). Is it possible that such MOF is caused by microcirculatory disturbances, resembling the pathogenesis of TTP.

Human immunodeficiency virus (HIV) infection is also a known trigger of TMAs (48). In our registry, only one HIVpositive patient with severe deficiency of ADAMTS13: AC with positive ADAMTS13: INH was identified.

Finally, the TMAs that fell into the miscellaneous subcategory are too variable to address in this report. The details of some of these patients will be reported in detail elsewhere by referral physicians.

\section{Acknowledgement}

The authors thank Dr. Masahito Uemura for his critical reading and valuable comments. We also thank Ms Ayami Isonishi, and Drs. Hideo Yagi and Hiromichi Ishizashi for their excellent technical assistance performing ADAMTS13: AC assays.

Grant Support: This work was supported in part by research grants from the Ministry of Education, Culture, Sports, Science and Technology of Japan and from the Ministry of Health, Labor, and Welfare of Japan for Blood Coagulation Abnormalities (Successive Directors; Drs. Masao Nakagawa, Yasuo Ikeda, and Mitsuru Murata), and by the reward of Erwin von Bälz prize in 2008 .

\section{References}

1. Moake JL. Thrombotic microangiopathies. N Engl J Med 347: 589-600, 2002.

2. George JN. Clinical practice. Thrombotic thrombocytopenic purpura. N Engl J Med 354: 1927-1935, 2006.

3. Furlan M, Robles R, Lammle B. Partial purification and characterization of a protease from human plasma cleaving von Willebrand factor to fragments produced by in vivo proteolysis. Blood 87: 4223-4234, 1996.

4. Tsai HM. Physiologic cleavage of von Willebrand factor by a plasma protease is dependent on its conformation and requires calcium ion. Blood 87: 4235-4244, 1996.

5. Gerritsen HE, Robles R, Lammle B, Furlan M. Partial amino acid sequence of purified von Willebrand factor-cleaving protease. Blood 98: 1654-1661, 2001.

6. Fujikawa K, Suzuki H, McMullen B, Chung D. Purification of human von Willebrand factor-cleaving protease and its identification as a new member of the metalloproteinase family. Blood $\mathbf{9 8}$ : 1662-1666, 2001

7. Soejima K, Mimura N, Hirashima M, et al. A novel human metalloprotease synthesized in the liver and secreted into the blood: possibly, the von Willebrand factor-cleaving protease? J Biochem (Tokyo) 130: 475-480, 2001.

8. Zheng X, Chung D, Takayama TK, Majerus EM, Sadler JE, Fujikawa K. Structure of von Willebrand factor-cleaving protease (ADAMTS13), a metalloprotease involved in thrombotic thrombocytopenic purpura. J Biol Chem 276: 41059-41063, 2001.

9. Uemura M, Tatsumi $\mathrm{K}$, Matsumoto $\mathrm{M}$, et al. Localization of
ADAMTS13 to the stellate cells of human liver. Blood 106: 922924, 2005.

10. Suzuki M, Murata M, Matsubara Y, et al. Detection of von Willebrand factor-cleaving protease (ADAMTS-13) in human platelets. Biochem Biophys Res Commun 313: 212-216, 2004.

11. Turner N, Nolasco L, Tao Z, Dong JF, Moake J. Human endothelial cells synthesize and release ADAMTS-13. J Thromb Haemost 4: 1396-1404, 2006.

12. Manea M, Kristoffersson A, Schneppenheim R, et al. Podocytes express ADAMTS13 in normal renal cortex and in patients with thrombotic thrombocytopenic purpura. Br J Haematol 138: 651662, 2007.

13. Furlan M, Robles R, Galbusera M, et al. von Willebrand factorcleaving protease in thrombotic thrombocytopenic purpura and the hemolytic-uremic syndrome. N Engl J Med 339: 1578-1584, 1998.

14. Tsai HM, Lian EC. Antibodies to von Willebrand factor-cleaving protease in acute thrombotic thrombocytopenic purpura. N Engl J Med 339: 1585-1594, 1998.

15. Sadler JE. Von Willebrand factor, ADAMTS13, and thrombotic thrombocytopenic purpura. Blood 112: 11-18, 2008.

16. Tandon NN, Rock G, Jamieson GA. Anti-CD36 antibodies in thrombotic thrombocytopenic purpura. Br J Haematol 88: 816825, 1994.

17. Davis AK, Makar RS, Stowell CP, Kuter DJ, Dzik WH. ADAMTS 13 binds to CD36: a potential mechanism for platelet and endothelial localization of ADAMTS13. Transfusion 49: 206-213, 2009. 
18. Febbraio M, Hajjar DP, Silverstein RL. CD36: a class B scavenger receptor involved in angiogenesis, atherosclerosis, inflammation, and lipid metabolism. J Clin Invest 108: 785-791, 2001.

19. Coppo P, Veyradier A. Thrombotic microangiopathies: towards a pathophysiology-based classification. Cardiovasc Hematol Disord Drug Targets 9: 36-50, 2009.

20. Kinoshita S, Yoshioka A, Park YD, et al. Upshaw-Schulman syndrome revisited: a concept of congenital thrombotic thrombocytopenic purpura. Int J Hematol 74: 101-108, 2001.

21. Kato S, Matsumoto M, Matsuyama T, Isonishi A, Hiura H, Fujimura Y. Novel monoclonal antibody-based enzyme immunoassay for determining plasma levels of ADAMTS13 activity. Transfusion 46: 1444-1452, 2006.

22. Mori $\mathrm{Y}$, Wada $\mathrm{H}$, Gabazza EC, et al. Predicting response to plasma exchange in patients with thrombotic thrombocytopenic purpura with measurement of $\mathrm{vWF}$-cleaving protease activity. Transfusion 42: 572-580, 2002.

23. Kasper CK, Aledort L, Aronson D, et al. Proceedings: A more uniform measurement of factor VIII inhibitors. Thromb Diath Haemorrh 34: 612, 1975.

24. Ho VT, Cutler C, Carter S, et al. Blood and marrow transplant clinical trials network toxicity committee consensus summary: thrombotic microangiopathy after hematopoietic stem cell transplantation. Biol Blood Marrow Transplant 11: 571-575, 2005.

25. Ruutu T, Barosi G, Benjamin RJ, et al. Diagnostic criteria for hematopoietic stem cell transplant-associated microangiopathy: results of a consensus process by an International Working Group. Haematologica 92: 95-100, 2007.

26. Wada H, Wakita $Y$, Nakase $T$, et al. Increased plasma-soluble fibrin monomer levels in patients with disseminated intravascular coagulation. Am J Hematol 51: 255-260, 1996.

27. Fujimura Y, Matsumoto M, Yagi H, Yoshioka A, Matsui T, Titani K. Von Willebrand factor-cleaving protease and Upshaw-Schulman syndrome. Int J Hematol 75: 25-34, 2002.

28. Kokame K, Aoyama Y, Matsumoto M, Fujimura Y, Miyata T. Inherited and de novo mutations of ADAMTS13 in a patient with Upshaw-Schulman syndrome. J Thromb Haemost 6: 213-215, 2008.

29. Medina PJ, Sipols JM, George JN. Drug-associated thrombotic thrombocytopenic purpura-hemolytic uremic syndrome. Curr Opin Hematol 8: 286-293, 2001.

30. Bennett CL, Kim B, Zakarija A, et al. Two mechanistic pathways for thienopyridine-associated thrombotic thrombocytopenic purpura: a report from the SERF-TTP Research Group and the RADAR Project. J Am Coll Cardiol 50: 1138-1143, 2007.

31. Fukusako T, Yamashita H, Omoto M, Matsuda K, Shinohara K, Fujimura Y. A case of thrombotic thrombocytopenic purpura associated with clopidogrel. Clin Neurol 47: 635-638, 2007.

32. Zakarija A, Kwaan HC, Moake JL, et al. Ticlopidine- and clopidogrel-associated thrombotic thrombocytopenic purpura (TTP): review of clinical, laboratory, epidemiological, and pharmacovigilance findings (1989-2008). Kidney Int 75 (Suppl 112): S20-S24, 2009.

33. Zakarija A, Bennett C. Drug-induced thrombotic microangiopathy. Semin Thromb Hemost 31: 681-690, 2005.
34. Kitano K, Gibo Y, Kamijo A, et al. Thrombotic thrombocytopenic purpura associated with pegylated-interferon alpha-2a by an ADAMTS13 inhibitor in a patient with chronic hepatitis C. Haematologica 91: ECR34, 2006.

35. Gitlow S, Goldmark C. Generalized capillary and arteriolar thrombosis. Report of two cases with a discussion of the literature. Ann Intern Med 13: 1046-1067, 1939.

36. Sato T, Hanaoka R, Ohshima M, et al. Analyses of ADAMTS13 activity and its inhibitor in patients with thrombotic thrombocytopenic purpura secondary to connective tissue diseases: Observations in a single hospital. Clin Exp Rheumatol 24: 454-455, 2006.

37. Matsuyama T, Kuwana M, Matsumoto M, Isonishi A, Inokuma S, Fujimura Y. Heterogeneous pathogenic processes of thrombotic microangiopathies in patients with connective tissue diseases. Thrombosis and Haemostasis 102: 371-378, 2009.

38. Kawahara M, Kanno M, Matsumoto M, Nakamura S, Fujimura Y, Ueno S. Diffuse neurodeficits in intravascular lymphomatosis with ADAMTS13 inhibitor. Neurology 63: 1731-1733, 2004.

39. Kanno $M$, Nakamura $S$, Kawahara $M$, et al. Chemotherapyresistant intravascular lymphoma accompanied by ADAMTS13 inhibitor successfully treated with rituximab. Int J Hematol 88: $345-$ 347, 2008.

40. van der Plas RM, Schiphorst ME, Huizinga EG, et al. von Willebrand factor proteolysis is deficient in classic, but not in bone marrow transplantation-associated, thrombotic thrombocytopenic purpura. Blood 93: 3798-3802, 1999.

41. Fujimura Y, Matsumoto M, Kokame K, et al. Pregnancy-induced thrombocytopenia and TTP, and the risk of fetal death, in Upshaw-Schulman syndrome: a series of 15 pregnancies in 9 genotyped patients. Br J Haematol 144: 742-754, 2009.

42. Uemura M, Fujimura $Y$, Matsumoto $M$, et al. Comprehensive analysis of ADAMTS13 in patients with liver cirrhosis. Thromb Haemost 99: 1019-1029, 2008.

43. Ko S, Okano E, Kanehiro H, et al. Plasma ADAMTS13 activity may predict early adverse events in living donor liver transplantation: observations in 3 cases. Liver Transpl 12: 859-869, 2006.

44. Kobayashi T, Wada H, Usui M, et al. Decreased ADAMTS13 levels in patients after living donor liver transplantation. Thromb Res 124: 541-545, 2009.

45. Pereboom IT, Adelmeijer J, van Leeuwen Y, Hendriks HG, Porte RJ, Lisman T. Development of a severe von Willebrand factor/ ADAMTS13 dysbalance during orthotopic liver transplantation. Am J Transplant 9: 1189-1196, 2009.

46. Wasserstein A, Hill G, Goldfarb S, Goldberg M. Recurrent thrombotic thrombocytopenic purpura after viral infection. Clinical and histologic simulation of chronic glomerulonephritis. Arch Intern Med 141: 685-687, 1981.

47. Dias PJ, Gopal S. Refractory thrombotic thrombocytopenic purpura following influenza vaccination. Anaesthesia 64: 444-446, 2009.

48. Bell WR, Chulay JD, Feinberg JE. Manifestations resembling thrombotic microangiopathy in patients with advanced human immunodeficiency virus (HIV) disease in a cytomegalovirus prophylaxis trial (ACTG 204). Medicine (Baltimore) 76: 369-380, 1997.

(C) 2010 The Japanese Society of Internal Medicine

http://www.naika.or.jp/imindex.html 